\title{
Splenic T Lymphoblastic Lymphoma
}

National Cancer Institute

\section{Source}

National Cancer Institute. Splenic T Lymphoblastic Lymphoma. NCI Thesaurus. Code C7311.

T lymphoblastic lymphoma that affects the spleen. 